\title{
A CLASSIFICATION OF NON-DEGENERATE HOMOGENEOUS EQUIAFFINE HYPERSURFACES IN FOUR COMPLEX DIMENSIONS*
}

\author{
MICHAEL EASTWOOD ${ }^{\dagger}$ AND VLADIMIR EZHOV ${ }^{\ddagger}$
}

\begin{abstract}
We solve the classification problem as in the title. We present explicit defining equations and give a characterization in terms of easily computable affine invariants.
\end{abstract}

1. Introduction. The equiaffine transformations of $\mathbb{C}^{4}$ are those of the form

$$
v \longmapsto A v+b \text { for } A \in \mathrm{SL}(4, \mathbb{C}) \text { and } b \in \mathbb{C}^{4} \text {. }
$$

A complex hypersurface $\Sigma \subset \mathbb{C}^{4}$ is said to be equiaffine homogeneous if and only if it is the orbit of a complex Lie subgroup of the group of all equiaffine transformations. Such a hypersurface is everywhere non-singular. We shall also suppose that $\Sigma$ is nondegenerate (at one point and hence, by homogeneity, at all points). In this article, we classify the non-degenerate equiaffine homogeneous hypersurfaces in $\mathbb{C}^{4}$ up to affine equivalence.

The corresponding classification problem for surfaces in $\mathbb{C}^{3}$ was solved by Nomizu and Sasaki [9]. There are six such surfaces:-

1. $Z=X Y$

2. $Z^{2}=X Y+1$

3. $X Y Z=1$

4. $X^{2}\left(Z+Y^{2}\right)^{3}=1$

5. $Z=X Y+X^{3}$

6. $Z=X Y+\log X$

The first five were found by Guggenheimer [5] and this list was completed in [9]. See [10] for further discussion and for general background on affine differential geometry.

In [1], the affine homogeneous surfaces in $\mathbb{C}^{3}$ were classified by means of normalizing their defining functions. The equiaffine cases have

1. $z=x y+\mathrm{O}(5)$

2. $z=x y+x^{2} y^{2}+\mathrm{O}(5)$

3. $z=x y+x^{3}+y^{3}+\mathrm{O}(5)$

4. $z=x y+x^{3}+y^{3}+\frac{9}{8} x^{4}-\frac{9}{2} x^{3} y-\frac{9}{4} x^{2} y^{2}-\frac{9}{2} x y^{3}+\frac{9}{8} y^{4}+\mathrm{O}(5)$

5. $z=x y+x^{3}+\mathrm{O}(5)$

6. $z=x y+x^{3}+x^{4}+\frac{16}{15} x^{5}+\mathrm{O}(6)$

as their normal forms. The higher order terms are determined by the given terms together with the requirement that the surface be affine homogeneous. In [1] this list is derived by choosing affine coördinates specially adapted to the surface and

\footnotetext{
*Received February 20, 2001; accepted for publication March 2, 2001.

†Department of Pure Mathematics, University of Adelaide, South AUSTRALIA 5005

(meastwoo@maths.adelaide.edu.au). Support from the Australian Research Council is gratefully acknowledged.

$\ddagger$ School of Mathematics, University of South Australia, Mawson Lakes, South AUSTRALIA 5095 (Vladimir.Ejov@unisa.edu.au).
} 
subject to criteria for homogeneity that restrict, order-by-order, the coefficients of the defining function. The point is that the first few terms of a defining function already determine the infinitesimal affine symmetries of a given surface. These symmetries form a Lie algebra called the symmetry algebra and exponentiation of the symmetry algebra determines the surface.

There is, however, a substantial difficulty in passing from $\mathbb{C}^{3}$ to $\mathbb{C}^{4}$. For surfaces in $\mathbb{C}^{3}$, the cubic terms of a defining function are easily normalized and carry very little information. In fact, the examples above are typical. As detailed in [1], the cubic terms $x^{3}+y^{3}$ signal a non-vanishing Pick invariant whilst if the Pick invariant is zero, then the cubic terms are absent or can be normalized to be $x^{3}$. By contrast, there is a great deal of affine invariant information at third order for a hypersurface in $\mathbb{C}^{4}$. It is possible to normalize the cubic terms but then the approach of [1] runs into trouble no matter what normalization is adopted. For the resulting equations to be tractable it seems that additional assumptions are needed. If, for example, the symmetry algebra is assumed to have dimension four or more, then additional normalizations may be adopted and the equations are easily solved [3]. Fortunately, once this case is eliminated, we may adopt a new approach that renders the resulting equations tractable.

This new approach utilizes directly the three-dimensional symmetry algebra. Lie algebras naturally divide into two classes. If the structure constants are trace-free, the Lie algebra is said to be Bianchi class $A$ and, otherwise, Bianchi class $B$ following Bianchi's classification of the three-dimensional Lie Algebras. In two dimensions there is, up to isomorphism, just one Lie algebra in each class. In three dimensions, the class B Lie algebras have canonical forms with sufficient structure to render the resulting equations tractable whilst for class A algebras normalizing the cubic terms of a defining equation works as before. So, this combination of techniques solves the problem. The classification is as follows.

THEOREM 1. The equiaffine homogeneous hypersurfaces in $\mathbb{C}^{4}$ are given locally by the following equations. Different equations and different values of the parameter $\alpha$ in A4 define affinely inequivalent hypersurfaces.

I1 $W=X Y+Z^{2}$

I2 $W^{2}=X Y+Z^{2}+1$

I3 $W=X Y+Z^{2}+X^{3}$

$14 W=X Y+Z^{2}+\log X$

I5 $W(X Y+Z)^{2}=1$

I6 $W^{2}\left(X Y+Z^{2}\right)^{3}=1$

A1 $W X Y Z=1$

A2 $W Z+W Y^{2}+X^{2} Z+X^{2} Y^{2}=1$

A3 $6 W X Y Z-4 X^{3} Z-3 X^{2} Y^{2}+W^{2} Z^{2}+4 W Y^{3}=1$

A4 $W=X Y+Z^{2}+X^{2} Z+\alpha X^{4}$

B1 $W Z^{2}=Z+X^{3}+X Y Z$

B2 $\left(W+X Y+X^{3}\right)^{2} Z=1$

B3 $\left(W Z+Y^{2}+X^{2} Z\right)^{4}=Z$

B4 $W^{2} X^{2}\left(Z+Y^{2}\right)^{3}=1$

B5 $\left(W+X Y+X^{2} Z\right)^{2} Z=1$

B6 $(W+X Y)^{2}\left(Z+X^{2}\right)=1$

B7 $W Z^{2}=Z+X^{2}+X Y Z$ 


$$
\begin{gathered}
\text { B8 }\left(W+Y Z+X^{2} Z\right)^{5}=Z \\
\text { B9 }\left(W Z+X^{2}+Y Z^{2}\right)^{5}=Z^{4} \\
\text { B10 } W=X Y+Z^{2}+X Z^{2} \\
\text { B11 } W^{2}=X Y+X^{2} Y+X^{2} Z
\end{gathered}
$$

In Section 2 we review Blaschke normal form for a defining function and the further normalizations that we shall adopt for the cubic terms in a defining equation. Also in this section are some canonical forms for matrices, useful in normalizing the symmetry algebra of a homogeneous hypersurface. In Section 3 it is explained how write down polynomial equations to capture that a hypersurface $\Sigma \subset \mathbb{C}^{4}$ be equiaffine homogeneous with three-dimensional symmetry algebra.

The main work is in Sections 4 and 5, which solve the equations derived in Section 3. Section 6 sorts out these solutions and those from [3] into a better list. In particular, we find easily computable invariants that distinguish between non-equivalent hypersurfaces.

The rest of the article comprises four appendices. The first two of these are concerned with the classical theory of the binary sextic and how it applies to the normalization and invariant theory we require. Several severe computations are required in our classification. For these we made extensive use of computer algebra. Rather than interrupt the main body of the article with continual references to computer programs, Appendix III is a commentary on these programs. The final appendix presents just one example of the explicit exponentiation of a symmetry algebra to determine the corresponding hypersurface.

Throughout this article, $\Sigma$ will always denote an equiaffine homogeneous hypersurface in $\mathbb{C}^{4}$. We shall use, without comment, the Einstein summation convention: a repeated index carries an implied sum over that index.

2. Normal Forms. We shall always choose coördinates $\left(u^{1}, u^{2}, u^{3}, w\right) \in \mathbb{C}^{4}$ so that $\Sigma$ passes through the origin and so that its tangent space at the origin is the hyperplane $\{w=0\}$. In other words, $\Sigma$ is defined by an equation

$$
w=F\left(u^{i}\right)=g_{i j} u^{i} u^{j}+a_{i j k} u^{i} u^{j} u^{k}+b_{i j k l} u^{i} u^{j} u^{k} u^{l}+\cdots .
$$

Non-degeneracy of $\Sigma$ means that the quadratic form $g_{i j}$ is non-degenerate. In addition, we shall suppose that the symmetric form $a_{i j k}$ is trace-free with respect to $g_{i j}$. It is shown in [6] that this requirement can always be met and that the remaining coördinate freedom is

$$
\left.\begin{array}{l}
w \mapsto r w \\
u^{i} \mapsto c^{i}{ }_{j} u^{j}
\end{array}\right\} \quad \text { for } r \neq 0 \text { and } c^{i}{ }_{j} \in \operatorname{GL}(3, \mathbb{C})
$$

This preferred $w$-axis is called the affine normal, a notion due to Blaschke in three dimensions. With $a_{i j k}$ trace-free we shall refer to (2.1) as Blaschke normal form. For further details, see [1].

Blaschke normal form applies to hypersurfaces in any dimension but from now on we shall specialize to hypersurfaces in four dimensions. In this case, we shall rename the $u^{i}$ coördinates as $x, y, z$ and normalize the quadratic form in (2.1) as follows:-

$$
g_{i j} u^{i} u^{j}=2 x y+z^{2} \text {. }
$$

Then $a_{i j k}$ being trace-free is to say that the cubic term $a_{i j k} u^{i} u^{j} u^{k}$ is a linear combination of the following monomials:-

$$
x^{3}, x^{2} z, x^{2} y-2 x z^{2}, 3 x y z-z^{3}, x y^{2}-2 y z^{2}, y^{2} z, y^{3} .
$$


The coördinate freedom (2.2) may further be used to normalize this linear combination whilst preserving (2.3). In Appendix I it is shown that, for non-zero cubic terms, the following normalizations may be adopted.

\begin{tabular}{|c|c|c|c|c|c|c|c|c|c|c|}
\cline { 2 - 11 } \multicolumn{1}{c|}{} & $\# 1$ & $\# 2$ & $\# 3$ & $\# 4$ & $\# 5$ & $\# 6$ & $\# 7$ & $\# 8$ & $\# 9$ & $\# 10$ \\
\hline$x^{3}$ & 0 & 0 & 0 & 0 & 0 & 0 & 0 & 0 & 0 & 1 \\
\hline$x^{2} z$ & 1 & 1 & 1 & 1 & 1 & 0 & 0 & 0 & 0 & 0 \\
\hline$x^{2} y-2 x z^{2}$ & $*$ & $*$ & $*$ & 1 & 0 & 1 & 1 & 1 & 0 & 0 \\
\hline $3 x y z-z^{3}$ & $*$ & $*$ & 1 & 0 & 0 & $*$ & 1 & 0 & 1 & 0 \\
\hline$x y^{2}-2 y z^{2}$ & $*$ & 1 & 0 & 0 & 0 & 1 & 0 & 0 & 0 & 0 \\
\hline$y^{2} z$ & 1 & 0 & 0 & 0 & 0 & 0 & 0 & 0 & 0 & 0 \\
\hline$y^{3}$ & 0 & 0 & 0 & 0 & 0 & 0 & 0 & 0 & 0 & 0 \\
\hline
\end{tabular}

Each column gives a possible normalization with *'s representing free parameters. Normal form \#1, for example, has 3 free parameters:

$$
w=2 x y+z^{2}+x^{2} z+a\left(x^{2} y-2 x z^{2}\right)+b\left(3 x y z-z^{3}\right)+c\left(x y^{2}-2 y z^{2}\right)+y^{2} z+\mathrm{O}(4)
$$

in its cubic terms. Note that Table (2.5) is not a classification. There are overlaps and repetitions under the action (2.2). For our purposes, it is only necessary to resolve this deficiency for the particular examples arising in our analysis of homogeneous surfaces. It is, however, worthwhile to note the general 8-fold multiplicity in normal form \#1 generated by the coördinate changes

$$
x \mapsto i x, y \mapsto-i y, z \mapsto-z \quad \text { and } \quad x \mapsto y, y \mapsto x, z \mapsto z .
$$

These changes generate the dihedral group $\mathfrak{D}_{4}$ and induce

$$
a \mapsto i a, b \mapsto-b, c \mapsto-i c \quad \text { and } \quad a \mapsto c, b \mapsto b, c \mapsto a .
$$

as regards the three parameters $a, b, c$.

The normalizations in Table (2.5) will be used in Section 4 in dealing with Bianchi Class A. Section 5 deals with Bianchi class B and then we shall need to normalize the Lie algebra itself. The following linear algebra is designed for this purpose.

Two real quadratic forms may be simultaneously diagonalized if one of them is definite. For indefinite or complex quadratic forms, this is no longer the case. The usual argument [11] involves eigenvectors and breaks down if an eigenvector turns out to be null. The proof of the following lemma is a modification of the usual argument and is left as an exercise.

LEMMA 1. Suppose that $H$ and $N$ are $3 \times 3$ symmetric complex matrices and that $H$ is non-degenerate. Then we can find an invertible $3 \times 3$ complex matrix $A$ such that

$$
A^{t} H A=\left[\begin{array}{lll}
0 & 1 & 0 \\
1 & 0 & 0 \\
0 & 0 & 1
\end{array}\right]
$$

and

$$
A^{t} N A=\left[\begin{array}{lll}
\nu & \lambda & 0 \\
\lambda & \nu & 0 \\
0 & 0 & \mu
\end{array}\right] \quad \text { or }\left[\begin{array}{lll}
0 & \lambda & 0 \\
\lambda & 1 & 0 \\
0 & 0 & \mu
\end{array}\right] \quad \text { or }\left[\begin{array}{ccc}
0 & \lambda & 0 \\
\lambda & 0 & 1 \\
0 & 1 & \lambda
\end{array}\right] \text {. }
$$


3. Setting up the Equations. The following procedure is fully explained in [3]. Also there is [2], where a similar story is told under the assumption of an Abelian symmetry algebra, and [1], which deals with homogeneous surfaces in affine threespace. We shall therefore be brief. For any polynomial $g(x, y, z)$, we shall write $\operatorname{Tr}^{N} g(x, y, z)$ for the truncated polynomial obtained by deleting terms of total degree greater than $N$.

THEOREM 2. Suppose $f(x, y, z)$ is a polynomial of degree $N$ without constant or linear terms. If $f(x, y, z)$ can be completed to a power series whose graph near the origin is an open subset of an affine homogeneous hypersurface $\Sigma$, then there are $4 \times 4$ matrices $P, Q, R$ such that

$$
\begin{aligned}
& \operatorname{Tr}{ }^{N-1}\left[\frac{\partial f}{\partial x}(x, y, z), \frac{\partial f}{\partial y}(x, y, z), \frac{\partial f}{\partial z}(x, y, z),-1\right] P\left[\begin{array}{c}
x \\
y \\
z \\
f(x, y, z)
\end{array}\right]=-\frac{\partial f}{\partial x}(x, y, z) \\
& \operatorname{Tr}^{N-1}\left[\frac{\partial f}{\partial x}(x, y, z), \frac{\partial f}{\partial y}(x, y, z), \frac{\partial f}{\partial z}(x, y, z),-1\right] Q\left[\begin{array}{c}
x \\
y \\
z \\
f(x, y, z)
\end{array}\right]=-\frac{\partial f}{\partial y}(x, y, z) \\
& \operatorname{Tr}^{N-1}\left[\frac{\partial f}{\partial x}(x, y, z), \frac{\partial f}{\partial y}(x, y, z), \frac{\partial f}{\partial z}(x, y, z),-1\right] R\left[\begin{array}{c}
x \\
y \\
z \\
f(x, y, z)
\end{array}\right]=-\frac{\partial f}{\partial z}(x, y, z) .
\end{aligned}
$$

Proof The proof is a simple modification of the corresponding result for surfaces proved in Theorem 1 and Corollary 1 of [1]. Suffice it to say that (3.1) constitutes the low order terms in the full defining equations for the symmetry algebra of $\Sigma$. That there are solutions is to say that there are infinitesimal symmetries in each of the three basic coördinate directions. This must be the case if $\Sigma$ is homogeneous.

When $N=3$, the system (3.1) is always soluble. Specifically, regarding $f(x, y, z)$ as fixed, (3.1) constitutes 27 linear equations which may be solved for 27 of the 48 entries in $P, Q$, and $R$. There are, however, other restrictions on $f(x, y, z)$ of degree 3 . They arise by considering what it means for the algebra generated by $P, Q$, and $R$ to be closed under Lie bracket as must be the case if we are to find a genuine symmetry algebra within the general solution of (3.1). To this end, let us write $P=\left(p_{i j}\right)$, $Q=\left(q_{i j}\right), R=\left(r_{i j}\right)$, and introduce quantities $m^{i j}$ for $1 \leq i, j \leq 3$ as follows:-

$$
\begin{array}{lll}
m^{11}=q_{13}-r_{12} & m^{12}=q_{23}-r_{22} & m^{13}=q_{33}-r_{32} \\
m^{21}=r_{11}-p_{13} & m^{22}=r_{21}-p_{23} & m^{23}=r_{31}-p_{33} \\
m^{31}=p_{12}-q_{11} & m^{32}=p_{22}-q_{21} & m^{33}=p_{32}-q_{31} .
\end{array}
$$

Theorem 3. Suppose $f(x, y, z)$ is a polynomial of degree 3 without constant or linear terms. Suppose $f(x, y, z)$ can be completed to a power series whose graph near the origin is an open subset of an equiaffine homogeneous hypersurface $\Sigma$ with threedimensional symmetry algebra. Then we can find a particular solution of (3.1) for $N=3$ with

$$
\text { trace } P=\operatorname{trace} Q=\operatorname{trace} R=0
$$

and

$$
\begin{aligned}
& Q R-R Q=m^{11} P+m^{12} Q+m^{13} R \\
& R P-P R=m^{21} P+m^{22} Q+m^{23} R \\
& P Q-Q P=m^{31} P+m^{32} Q+m^{33} R
\end{aligned}
$$


Conversely, for each such solution, $f(x, y, z)$ can be completed to a power series whose graph near the origin is an open subset of an equiaffine homogeneous hypersurface.

Proof The equations (3.3) restrict attention to equiaffine. symmetries. Then, the equations (3.4) say that the equiaffine symmetry algebra of $\Sigma$ is closed under Lie bracket. Once the symmetry algebra has closed in this way, the higher order terms in the power series expansion of the defining function are determined by exponentiating to the Lie subgroup whose orbit is $\Sigma$.

For equations (3.4) to hold we are supposing that $\Sigma$ is the orbit of a three-dimensional Lie subgroup. In general, the isotropy algebra consists of the infinitesimal symmetries of $\Sigma$ that also fix the origin. To order 3 , it gives rise to the subspace $\mathcal{I}$ consisting of $4 \times 4$ matrices $X$ satisfying the following system of linear equations:-

$$
\operatorname{Tr}^{3}\left[\frac{\partial f}{\partial x}(x, y, z), \frac{\partial f}{\partial y}(x, y, z), \frac{\partial f}{\partial z}(x, y, z),-1\right] X\left[\begin{array}{c}
x \\
y \\
z \\
f(x, y, z)
\end{array}\right]=0 .
$$

and we require only that (3.4) hold modulo $\mathcal{I}$. Complete details in the analogous case of homogeneous surfaces in $\mathbb{C}^{3}$ are given in [1].

When the symmetry algebra is three-dimensional, the quantities $m^{i j}$ introduced in (3.2) may be viewed as its structure constants. This is clearly exhibited in (3.4). In particular, if we introduce

$$
v_{1}=m^{23}-m^{32} \quad v_{2}=m^{31}-m^{13} \quad v_{3}=m^{12}-m^{21},
$$

then the Jacobi identities read

$$
v_{i} m^{i j}=0 \text { for } j=1,2,3 .
$$

It is convenient to regard $v=\left(v_{i}\right)$ as a row vector and $M=\left(m^{i j}\right)$ as a $3 \times 3$ matrix. Then (3.7) reads $v M=0$. The proof of the following lemma is elementary and will be omitted.

LEMMA 2. If we write the quantities $c^{i}{ }_{j}$ in (2.2) and $g_{i j}$ in (2.1) as matrices $C=\left(c^{i}{ }_{j}\right)$ and $G=\left(g_{i j}\right)$, then

$$
G \mapsto \frac{1}{r} C^{t} G C \quad M \mapsto \operatorname{det}(C) C^{-1} M\left(C^{-1}\right)^{t} \quad v \mapsto v C
$$

under the change of coördinates (2.2).

This suggests that we split $M$ into its symmetric and skew parts, such a splitting being preserved under (3.8). The vector $v$ is, in effect, the skew part so let us write $\left(n^{i j}\right)=N=\left(M+M^{t}\right) / 2$ for the symmetric part. The Jacobi identity now reads $v N=0$. This is the only constraint in order that the quantities $m^{i j}$ define a lie algebra.

DeFINITION 1. We shall say that the symmetry algebra defined by $m^{i j}$ is Bianchi class $A$ if and only if $v=0$. Otherwise, we shall say that it is Bianchi class $B$.

In summary, we have three systems of equations in the 48 variables $p_{i j}, q_{i j}$, and $r_{i j}$ in order that $f(x, y, z)$ of degree 3 can be completed to define an equiaffine homogeneous hypersurface. Firstly, there are the 27 linear equations (3.1). Each line of (3.1) is an equality of second degree polynomials without constant term in $x, y, z$ and so each may be regarded as 9 equations for the 9 coefficients of $x, y, z, x^{2}, y^{2}, z^{2}, x y, y z, z x$. For equiaffine hypersurfaces we have 3 further linear equations (3.3). Finally there is a system of quadratic equations (3.4). All these equations must be satisfied in the case of an equiaffine homogeneous hypersurface with three-dimensional symmetry algebra. 
It is easy to check (with computer algebra) that the linear system (3.1) may be uniquely solved for the 27 variables

$\begin{array}{lllllllll}p_{11} & p_{21} & p_{31} & p_{41} & p_{42} & p_{43} & q_{13} & q_{23} & q_{33} \\ q_{12} & q_{22} & q_{32} & q_{41} & q_{42} & q_{43} & r_{11} & r_{21} & r_{31} \\ r_{13} & r_{23} & r_{33} & r_{41} & r_{42} & r_{43} & p_{12} & p_{22} & p_{32}\end{array}$

and (3.3) for the 3 variables

$p_{44} \quad q_{44} \quad r_{44}$.

We may now eliminate

$$
\begin{array}{lllllllll}
r_{12} & r_{22} & r_{32} & p_{13} & p_{23} & p_{33} & q_{11} & q_{21} & q_{31}
\end{array}
$$

in favour of $m^{i j}$ by means of (3.2). Splitting $M=\left(m^{i j}\right)$ into its symmetric and skew parts, as above, we are left with a system (3.4) of quadratic equations in the 18 variables

$$
\begin{array}{ccccccccc}
p_{14} & p_{24} & p_{34} & q_{14} & q_{24} & q_{34} & r_{14} & r_{24} & r_{34} \\
v_{1} & v_{2} & v_{3} & n^{11} & n^{22} & n^{33} & n^{12} & n^{23} & n^{31}
\end{array}
$$

This is the system of equations we must solve.

4. Solving the Equations: Bianchi Class A. Let us adopt normal form \#1 from Table (2.5) for the cubic terms of a prospective defining equation. Thus, we take $f(x, y, z)$ to be

$$
2 x y+z^{2}+x^{2} z+a\left(x^{2} y-2 x z^{2}\right)+b\left(3 x y z-z^{3}\right)+c\left(x y^{2}-2 y z^{2}\right)+y^{2} z
$$

and consider the system (3.4) in the variables (3.9). Assuming that the symmetry algebra is Bianchi class A, we may set

$$
v_{1}=v_{2}=v_{3}=0
$$

Regarding the parameters $a, b, c$ as additional variables it is now possible (with computer algebra) to solve the resulting system. Taking into account the general 8-fold multiplicity (2.6), there are just five possibilities for $a, b, c$ as follows.

\begin{tabular}{|c||c|c|c|c|c|}
\hline$a$ & 0 & -1 & 0 & $\sqrt{3 / 2}$ & $(i+1) / 2$ \\
\hline$b$ & 0 & -1 & $2 / 3$ & $-4 / 3$ & 0 \\
\hline$c$ & 0 & 1 & 0 & $-\sqrt{3 / 2}$ & $(i-1) / 2$ \\
\hline
\end{tabular}

In fact, the first two are equivalent. The linear coördinate change

$$
w \mapsto-4 w \quad\left[\begin{array}{l}
x \\
y \\
z
\end{array}\right] \mapsto\left[\begin{array}{ccc}
1+i & 1-i & -2 i \\
0 & -2+2 i & 0 \\
0 & -2+2 i & 2 i
\end{array}\right]\left[\begin{array}{l}
x \\
y \\
z
\end{array}\right]
$$

transforms the equation

$$
w=2 x y+z^{2}+x^{2} z-\left(x^{2} y-2 x z^{2}\right)-\left(3 x y z-z^{3}\right)+\left(x y^{2}-2 y z^{2}\right)+y^{2} z+\mathrm{O}(4)
$$

into $w=2 x y+z^{2}+x^{2} z+y^{2} z+\mathrm{O}(4)$. Similarly, the last three are all equivalent and may be placed in the more convenient normal form $w=2 x y+z^{2}+x^{3}+y^{3}+\mathrm{O}(4)$ by 
means of the following coördinate changes:-

$$
\begin{array}{ll}
w \mapsto \frac{9}{8} w & {\left[\begin{array}{l}
x \\
y \\
z
\end{array}\right] \mapsto \frac{3}{8}\left[\begin{array}{ccc}
-\sqrt{2} i & \sqrt{2} i & -2 i \\
-\sqrt{2} i & \sqrt{2} i & 2 i \\
-2 & -2 & 0
\end{array}\right]\left[\begin{array}{l}
x \\
y \\
z
\end{array}\right]} \\
w \mapsto 18 w & {\left[\begin{array}{l}
x \\
y \\
z
\end{array}\right] \mapsto \frac{3}{2}\left[\begin{array}{ccc}
-2 \sqrt{2} i & 2 \sqrt{2} i & -4 i \\
\sqrt{2}(\sqrt{3}+i) & \sqrt{2}(\sqrt{3}-i) & 4 i \\
-2-2 \sqrt{3} i & -2+2 \sqrt{3} i & -2 \sqrt{6} i
\end{array}\right]\left[\begin{array}{l}
x \\
y \\
z
\end{array}\right]} \\
w \mapsto-2 w & {\left[\begin{array}{l}
x \\
y \\
z
\end{array}\right] \mapsto \frac{1}{\sqrt{3}}\left[\begin{array}{ccc}
-1-i & 1+i & -\sqrt{2}(1+i) \\
(1-i) \omega & -(1-i) \omega^{2} & \sqrt{2}(1-i) \\
2 \omega^{2} & -2 \omega & -\sqrt{2}
\end{array}\right]\left[\begin{array}{l}
x \\
y \\
z
\end{array}\right]}
\end{array}
$$

where $\omega=\exp (2 \pi i / 3)$. Setting up (3.4) for cubic terms normalized as \#2, \#3, $\# 4, \# 6$, \#7, \#8, or \#9 gives an inconsistent system of equations in (3.9) and the parameters of the normal form, if any.

Therefore, to finish our analysis of Bianchi class A symmetry algebras, it suffices to impose (4.1) and solve (3.4) in the variables (3.9) with $f(x, y, z)$ one of the following five possibilities:-

Case 1: $f(x, y, z)=2 x y+z^{2}+x^{2} z+y^{2} z$

Case 2: $\quad f(x, y, z)=2 x y+z^{2}+x^{3}+y^{3}$

Case 3: $\quad f(x, y, z)=2 x y+z^{2}+x^{2} z$

Case 4: $\quad f(x, y, z)=2 x y+z^{2}+x^{3}$

Case 5: $f(x, y, z)=2 x y+z^{2}$.

This is easily done (with computer algebra). In cases $1-4$, we find that

$$
p_{34}=q_{34}=r_{14}=r_{24}=n_{23}=n_{31}=0
$$

and the remaining variables are as follows

\begin{tabular}{|c|c|c|c|c|c|c|c|c|c|}
\cline { 2 - 10 } \multicolumn{1}{c|}{} & $p_{14}$ & $p_{24}$ & $q_{14}$ & $q_{24}$ & $r_{34}$ & $n_{11}$ & $n_{22}$ & $n_{33}$ & $n_{12}$ \\
\hline \multirow{4}{*}{ Case 1 } & $1 / 4$ & $\pm i / 4$ & $\mp i / 4$ & $1 / 4$ & 0 & $-1 / 4$ & $1 / 4$ & $\mp i / 2$ & $\pm i / 4$ \\
\cline { 2 - 10 } & $1 / 8$ & 0 & 0 & $1 / 8$ & $1 / 8$ & 0 & 0 & 0 & 0 \\
\cline { 2 - 10 } & 0 & 0 & 0 & 0 & $1 / 2$ & $1 / 2$ & $-1 / 2$ & 0 & 0 \\
\hline Case 2 & $27 / 64$ & 0 & 0 & $27 / 64$ & $27 / 64$ & 0 & 0 & $\pm \sqrt{27 / 8}$ & $\pm \sqrt{3 / 8}$ \\
\hline Case 3 & 0 & 0 & 0 & 0 & 0 & 0 & $\beta$ & 0 & 0 \\
\hline Case 4 & 0 & 0 & 0 & 0 & 0 & 0 & $\beta$ & 0 & 0 \\
\hline
\end{tabular}

where $\beta$ is an arbitrary parameter. Complete details are included in the computer prgrams described in Appendix III.

In each case, the right hand sides of (3.1) with $N=4$ determine the quartic terms in the corresponding power series. In case 1, for example, we obtain the following possible continuations:-

$$
\begin{aligned}
& w=2 x y+z^{2}+x^{2} z+y^{2} z+\frac{1}{16}(x \mp i y)^{4} \mp \frac{i}{2}(x \pm i y)^{2} z^{2}+\mathrm{O}(5) \\
& w=2 x y+z^{2}+x^{2} z+y^{2} z+\frac{1}{8} x^{4}+\frac{1}{8} y^{4}+\frac{3}{4} x y z^{2}-\frac{1}{16} z^{4}+\mathrm{O}(5) \\
& w=2 x y+z^{2}+x^{2} z+y^{2} z+\frac{1}{4} x^{4}+\frac{1}{2} x^{2} y^{2}+\frac{1}{4} y^{4}-\frac{1}{4} z^{4}+\mathrm{O}(5) .
\end{aligned}
$$


Futhermore, if we now regard (3.1) with $N=4$ as linear equations for the matrices $P, Q, R$, then each of these continuations determines, already at fourth order, the symmetry algebra and hence the hypersurface itself. Thus, we only need to know these defining functions up to fourth order to fix the hypersurface. In fact, up to affine equivalence, there are only two hypersurfaces here. The coördinate change

$$
x \mapsto i x, y \mapsto-i y, z \mapsto-z
$$

effects the changes of sign in the first equation and

$$
\left[\begin{array}{l}
x \\
y \\
z
\end{array}\right] \mapsto \frac{1}{2}\left[\begin{array}{ccc}
-i & -1 & 1-i \\
-1 & i & 1+i \\
-1+i & -1-i & 0
\end{array}\right]\left[\begin{array}{l}
x \\
y \\
z
\end{array}\right]
$$

transforms

$$
2 x y+z^{2}+x^{2} z+y^{2} z+\frac{1}{16}(x-i y)^{4}-\frac{i}{2}(x+i y)^{2} z^{2}
$$

into

$$
2 x y+z^{2}+x^{2} z+y^{2} z+\frac{1}{4} x^{4}+\frac{1}{2} x^{2} y^{2}+\frac{1}{4} y^{4}-\frac{1}{4} z^{4} .
$$

In case 2 we obtain

$$
w=2 x y+z^{2}+x^{3}+y^{3}+\frac{45}{32} x^{2} y^{2} \pm \sqrt{\frac{27}{32}} x^{3} z \mp \sqrt{\frac{27}{32}} y^{3} z-\frac{27}{32} x y z^{2}-\frac{27}{128} z^{4}+\mathrm{O}(5)
$$

Again, the hypersurface is determined already by these quartic terms and the signs are an artefact of choice of coördinates. In case 3 we obtain

$$
w=2 x y+z^{2}+x^{2} z+\frac{1}{8}(1-2 \beta) x^{4}+\mathrm{O}(5)
$$

In case 4 , the parameter $\beta$ does not show up in the continuation:-

$$
w=2 x y+z^{2}+x^{3}+\mathrm{O}(5) .
$$

In fact, this case has non-trivial isotropy and the parameter $\beta$ is absorbed into this isotropy. In any case, having an equiaffine symmetry algebra of dimension greater than three is grounds for exclusion. Case 5 also gives nothing of interest: there are two possible continuations but both have isotropy.

The following theorem summarises the results obtained in this Section.

THEOREM 4. Suppose $\Sigma$ is an equiaffine homogeneous hypersurface in $\mathbb{C}^{4}$ with three-dimensional symmetry algebra of Bianchi class $A$. Then we may choose affine coördinates to render $\Sigma$ in one of the following normal forms:-

$$
\begin{aligned}
\text { A1 } w= & 2 x y+z^{2}+x^{2} z+y^{2} z+\frac{1}{8} x^{4}+\frac{1}{8} y^{4}+\frac{3}{4} x y z^{2}-\frac{1}{16} z^{4}+\mathrm{O}(5) \\
\text { A2 } w= & 2 x y+z^{2}+x^{2} z+y^{2} z+\frac{1}{4} x^{4}+\frac{1}{2} x^{2} y^{2}+\frac{1}{4} y^{4}-\frac{1}{4} z^{4}+\mathrm{O}(5) \\
\text { A3 } w= & 2 x y+z^{2}+x^{3}+y^{3} \\
& \quad+\frac{45}{32} x^{2} y^{2}+\sqrt{\frac{27}{32}} x^{3} z-\sqrt{\frac{27}{32}} y^{3} z-\frac{27}{32} x y z^{2}-\frac{27}{128} z^{4}+\mathrm{O}(5)
\end{aligned}
$$

A4 $w=2 x y+z^{2}+x^{2} z+\alpha x^{4}+\mathrm{O}(5)$.

In each case, the higher order terms in a convergent power series are determined by its truncation at fourth order as given.

In Section 6 we shall see that these normal forms are distinct. 
5. Solving the Equations: Bianchi Class B. In this section we shall adopt Blaschke normal form (2.1) for the defining function but make no further normalizations of its cubic terms. Instead, we shall spend the remaining coördinate freedom (2.2) in normalizing its quadratic terms and the symmetry algebra. Lemma 1 is designed for this purpose. Recall that $N$ is the symmetric part of $M$, the matrix of structure constants of our symmetry algebra. Recall that $G=\left(g_{i j}\right)$ represents the quadratic terms in the defining equation and let $H=G^{-1}$. Lemma 2 says how these matrices transform under (2.2). Thus, we can normalize the quadratic terms (2.3) and arrange that $N$ be in one of the canonical forms (2.7). This puts $N G$ into one of the following canonical forms:-

\begin{tabular}{|c|c|c|c|} 
Segre characteristic & {$[111]$} & {$[21]$} & {$[3]$} \\
\hline \multirow{N}{*}{$\mathrm{NG}$} & {$\left[\begin{array}{lll}\lambda & \nu & 0 \\
\nu & \lambda & 0 \\
0 & 0 & \mu\end{array}\right]$} & {$\left[\begin{array}{ccc}\lambda & 0 & 0 \\
1 & \lambda & 0 \\
0 & 0 & \mu\end{array}\right]$} & {$\left[\begin{array}{ccc}\lambda & 0 & 0 \\
0 & \lambda & 1 \\
1 & 0 & \lambda\end{array}\right] \mid$}
\end{tabular}

As in [11], the Segre characteristic records the arrangement of blocks in the Jordan canonical form. It is preserved under (3.8).

We may also normalize $v$. Consider, for example, the case of Segre characteristic [21]. The form of $v$ is limited by Jacobi identity $v N=0$. One possibility is that $\mu=0$ and $v=[0,0, h]$ for some non-zero $h$. In this case, by executing the coördinate change

$$
w \mapsto \frac{1}{h^{2}} w \quad\left[\begin{array}{l}
x \\
y \\
z
\end{array}\right] \mapsto\left[\begin{array}{ccc}
1 / \sqrt{h} & 0 & 0 \\
0 & 1 / h \sqrt{h} & 0 \\
0 & 0 & 1 / h
\end{array}\right]\left[\begin{array}{l}
x \\
y \\
z
\end{array}\right]
$$

and redefining $\lambda$, we find that $h$ may be normalized to unity. Similar reasoning in other cases yields the following canonical forms

\begin{tabular}{c|c|c|c|c|} 
& {$[111]$} & {$[21]$ type 1} & {$[21]$ type 2} & {$[3]$} \\
\hline $\mathrm{N}$ & {$\left[\begin{array}{lll}\nu & \lambda & 0 \\
\lambda & \nu & 0 \\
0 & 0 & 0\end{array}\right]$} & {$\left[\begin{array}{lll}0 & \lambda & 0 \\
\lambda & 1 & 0 \\
0 & 0 & 0\end{array}\right]$} & {$\left[\begin{array}{ccc}0 & 0 & 0 \\
0 & 1 & 0 \\
0 & 0 & \mu\end{array}\right]$} & {$\left[\begin{array}{ccc}0 & 0 & 0 \\
0 & 0 & 1 \\
0 & 1 & 0\end{array}\right]$} \\
\hline $\mathrm{v}$ & {$[0,0,1]$} & {$[0,0,1]$} & {$[1,0,0]$} & {$[h, 0,0]$}
\end{tabular}

whilst maintaining (2.3) for the quadratic terms. Note that when the Segre characteristic is [3], the non-zero parameter $h$ cannot be eliminated.

To analyze Bianchi class B then, we construct the system (3.4) for

$$
f(x, y, z)=2 x y+z^{2}+\text { an arbitrary linear combination of }(2.4)
$$

and solve for the coefficients of this linear combination and the variables (3.9) normalized as above. This is easily done (with computer algebra). The details are contained in the programs discussed in Appendix III. The results are as follows.

Segre characteristic [111]. There are 14 possible solutions 6 of which have isotropy and may, therefore, be discarded. The remaining 8 occur in pairs under the 
change of coördinates $x \leftrightarrow y$. This leaves 4 possible hypersurfaces:-

$$
\begin{aligned}
& w=2 x y+z^{2}+\alpha x^{3}-\frac{1}{4} x^{2} y^{2}-\frac{1}{2} \alpha x^{3} z-\frac{1}{4} x y z^{2}-\frac{1}{16} z^{4}-\frac{1}{2} \alpha x^{4} y+\mathrm{O}(6) \\
& w=2 x y+z^{2}+\alpha x^{3}+2 x y z-\frac{2}{3} z^{3}+x^{2} y^{2}+\alpha x^{3} z-2 x y z^{2}+\mathrm{O}(5) \\
& w=2 x y+z^{2}+\frac{2}{5} i x^{2} z+\frac{2}{5} x y z-\frac{2}{15} z^{3}-\frac{2}{5} i y^{2} z+\frac{6}{25} i x^{3} y-\frac{3}{25} x^{2} y^{2} \\
& -\frac{6}{25} i x y^{3}+\frac{1}{25} i x^{2} z^{2}-\frac{8}{25} x y z^{2}-\frac{1}{25} i y^{2} z^{2}-\frac{1}{10} z^{4}+\mathrm{O}(5) \\
& w=2 x y+z^{2}+\frac{5}{2} i x^{2} z-x y z+\frac{1}{3} z^{3}-\frac{5}{2} i y^{2} z-\frac{35}{32} x^{4}+\frac{5}{8} i x^{3} y+\frac{9}{16} x^{2} y^{2} \\
& -\frac{5}{8} i x y^{3}-\frac{35}{32} y^{4}-\frac{5}{8} i x^{2} z^{2}+\frac{13}{4} x y z^{2}+\frac{5}{8} i y^{2} z^{2}-\frac{5}{8} z^{4}+\mathrm{O}(5) .
\end{aligned}
$$

The first of these is unusual in that the fifth order terms must be given before the symmetry algebra closes. The parameter $\alpha$ in the first two power series is not invariant:-

$$
x \mapsto \lambda x \quad y \mapsto \lambda^{-1} y \quad \text { induces } \quad \alpha \mapsto \lambda^{3} \alpha .
$$

Thus, if $\alpha$ is non-zero, it may be rescaled to unity. On the other hand, if $\alpha$ vanishes, then (5.1) generates isotropy so the hypersurface may be discarded.

Segre characteristic [21] type 1 . There are 6 possible solutions 3 of which have isotropy and may, therefore, be discarded. This leaves 3 possible hypersurfaces:-

$$
\begin{aligned}
w=2 x y+z^{2} & +2 x^{2} z+2 x y z-\frac{2}{3} z^{3} \\
& +2 x^{3} y+x^{2} y^{2}+3 x^{2} z^{2}-2 x y z^{2}+\mathrm{O}(5) \\
w=2 x y+z^{2}- & 2 x^{2} z+2 x y z-\frac{2}{3} z^{3} \\
+ & x^{4}-2 x^{3} y+x^{2} y^{2}+2 x^{2} z^{2}-2 x y z^{2}+\mathrm{O}(5) \\
w=2 x y+z^{2} & +2 x^{2} z+x^{3} y-\frac{1}{4} x^{2} y^{2}-\frac{1}{2} x^{2} z^{2}-\frac{1}{4} x y z^{2}-\frac{1}{16} z^{4}+\mathrm{O}(5) .
\end{aligned}
$$

Segre characteristic [21] type 2. There is just one solution to (3.4) but it has isotropy and may, therefore, be discarded.

Segre characteristic [3]. There are 3 possible solutions 1 of which has isotropy and may, therefore, be discarded. This leaves 2 possible hypersurfaces:-

$$
\begin{aligned}
& w=2 x y+z^{2}+\alpha x^{3}+2 x^{2} y-4 x z^{2}+\frac{9}{2} \alpha x^{4}+2 x^{3} y-4 x^{2} z^{2}+\mathrm{O}(5) \\
& w=2 x y+z^{2}+\alpha x^{3}-\frac{4}{3} x^{2} y+\frac{8}{3} x z^{2}+2 \alpha x^{4}+\frac{8}{9} x^{3} y+\frac{28}{3} x^{2} z^{2}+\mathrm{O}(5) .
\end{aligned}
$$

The parameter $\alpha$ is not invariant:-

$$
w \mapsto \lambda^{2} w \quad y \mapsto \lambda^{2} y \quad z \mapsto \lambda z \quad \text { induces } \quad \alpha \mapsto \lambda^{-2} \alpha .
$$

Thus, if $\alpha$ is non-zero, it may be rescaled to unity. So, these cases split according to whether $\alpha$ vanishes. We obtain a total of 4 possible hypersurfaces.

Bianchi class B summary. The following theorem gathers the hypersurfaces obtained above. For convenience somę elementary coördinate rescalings have been employed.

THEOREM 5. Suppose $\Sigma$ is an equiaffine homogeneous hypersurface in $\mathbb{C}^{4}$ with three-dimensional symmetry algebra of Bianchi class B. Then we may choose affine coördinates to render $\Sigma$ in one of the following normal forms:- 


$$
\begin{aligned}
& \text { B1 } w=2 x y+z^{2}+x^{3}-4 x^{2} y^{2}-2 x^{3} z-4 x y z^{2}-z^{4}-8 x^{4} y+\mathrm{O}(6) \\
& \text { B2 } w=2 x y+z^{2}+x^{3}+3 x y z-z^{3}+\frac{9}{4} x^{2} y^{2}+\frac{3}{2} x^{3} z-\frac{9}{2} x y z^{2}+\mathrm{O}(5) \\
& \text { B3 } w=2 x y+z^{2}+2 x^{2} z+2 x y z-\frac{2}{3} z^{3}+2 y^{2} z+6 x^{3} y \\
& -3 x^{2} y^{2}+6 x y^{3}+x^{2} z^{2}-8 x y z^{2}+y^{2} z^{2}-\frac{5}{2} z^{4}+\mathrm{O}(5) \\
& \text { B4 } w=2 x y+z^{2}+5 x^{2} z+2 x y z-\frac{2}{3} z^{3}+5 y^{2} z \\
& +\frac{35}{8} x^{4}-\frac{5}{2} x^{3} y+\frac{9}{4} x^{2} y^{2}-\frac{5}{2} x y^{3}+\frac{35}{8} y^{4} \\
& +\frac{5}{2} x^{2} z^{2}+13 x y z^{2}+\frac{5}{2} y^{2} z^{2}-\frac{5}{2} z^{4}+\mathrm{O}(5) \\
& \text { B5 } w=2 x y+z^{2}+x^{2} z+3 x y z-z^{3} \\
& +\frac{3}{2} x^{3} y+\frac{9}{4} x^{2} y^{2}+\frac{9}{4} x^{2} z^{2}-\frac{9}{2} x y z^{2}+\mathrm{O}(5) \\
& \text { B6 } w=2 x y+z^{2}+x^{2} z+3 x y z-z^{3} \\
& +\frac{1}{4} x^{4}+\frac{3}{2} x^{3} y+\frac{9}{4} x^{2} y^{2}-\frac{3}{2} x^{2} z^{2}-\frac{9}{2} x y z^{2}+\mathrm{O}(5) \\
& \text { B7 } w=2 x y+z^{2}+x^{2} z+2 x^{3} y-4 x^{2} y^{2}-x^{2} z^{2}-4 x y z^{2}-z^{4}+\mathrm{O}(5) \\
& \text { B8 } w=2 x y+z^{2}+x^{3}+x^{2} y-2 x z^{2}+\frac{9}{4} x^{4}+\frac{1}{2} x^{3} y-x^{2} z^{2}+\mathrm{O}(5) \\
& \text { B9 } w=2 x y+z^{2}+x^{3}+x^{2} y-2 x z^{2}-\frac{3}{2} x^{4}+\frac{1}{2} x^{3} y+\frac{21}{4} x^{2} z^{2}+\mathrm{O}(5) \\
& \mathbf{B 1 0} w=2 x y+z^{2}+x^{2} y-2 x z^{2}+\frac{1}{2} x^{3} y-x^{2} z^{2}+\mathrm{O}(5) \\
& \text { B11 } w=2 x y+z^{2}+x^{2} y-2 x z^{2}+\frac{1}{2} x^{3} y+\frac{21}{4} x^{2} z^{2}+\mathrm{O}(5) \text {. }
\end{aligned}
$$

In each case, the higher order terms in a convergent power series are determined by its truncation as given.

In the following section we shall prove that these normal forms are affinely inequivalent.

6. Interpreting the Solutions. Firstly, we deal with the possibility that $\Sigma$ has isotropy. In [3] the non-degenerate affine homogeneous hypersurfaces in $\mathbb{C}^{4}$ with isotropy are classified. It is an elementary matter to go through the classification of [3] (using the computer programs described therein) and pick out those that are equiaffine homogeneous with equiaffine isotropy. There are just six such hypersurfaces:-

THEOREM 6. Suppose $\Sigma$ is an equiaffine homogeneous hypersurface in $\mathbb{C}^{4}$ with equiaffine symmetry algebra of dimension greater than three. Then we may choose affine coördinates to render $\Sigma$ in one of the following affinely inequivalent normal forms:-

$$
\begin{array}{ll}
\text { I1 } & w=2 x y+z^{2}+\mathrm{O}(5) \\
\text { I2 } & w=2 x y+z^{2}+4 x^{2} y^{2}+4 x y z^{2}+z^{4}+\mathrm{O}(5) \\
\text { I3 } & w=2 x y+z^{2}+x^{3}+\mathrm{O}(5) \\
\text { I4 } & w=2 x y+z^{2}+x^{3}+x^{4}+\mathrm{O}(5) \\
\text { I5 } & w=2 x y+z^{2}+3 x y z-z^{3}+\frac{9}{4} x^{2} y^{2}-\frac{9}{2} x y z^{2}+\mathrm{O}(5) \\
\text { I6 } & w=2 x y+z^{2}+3 x y z-z^{3}-\frac{9}{16} x^{2} y^{2}-\frac{27}{16} x y z^{2}+\frac{45}{64} z^{4}+\mathrm{O}(5) .
\end{array}
$$

In each case, the higher order terms in a convergent power series are determined by its truncation at fourth order as given.

The explicit defining functions in [3] are reproduced in Theorem 1.

Next we deal with the class A hypersurfaces of Theorem 4. Appendix II attaches local affine invariants to every hypersurface, easily computable from the cubic terms of any defining equation. Their values on the class A homogeneous hypersurfaces are 
as follows:-

\begin{tabular}{|l|lll|}
\cline { 2 - 4 } \multicolumn{1}{c|}{} & \multicolumn{2}{l|}{ Cubic Invariants } & Remarks \\
\hline A1 & $j=\frac{1}{3} \quad k=\frac{1}{9} \quad l=0$ & Abelian \\
\hline A2 & $j=\frac{1}{3} \quad k=\frac{1}{9} \quad l=0$ & Non-Abelian \\
\hline A3 & $j=\frac{1}{2} \quad k=\frac{1}{4} \quad l=0$ & \\
\hline A4 & $I=J=K=L=0$ & Parameter $\alpha$ \\
\hline
\end{tabular}

The remarks concerning hypersurfaces $\mathbf{A 1}$ and $\mathbf{A 2}$ are directed at their symmetry algebras. From this table, it is clear that cases A1-A4 are affinely inequivalent and it remains to show that the parameter $\alpha$ in the hypersurface $\mathbf{A} 4$ is a genuine affine invariant. We remark in passing that the symmetry algebra of A4 is Abelian if and only if $\alpha=1 / 8$. Though all classical scalar invariants vanish, there is a non-vanishing vector-valued cubic covariant, namely

$$
S_{i j k l} \equiv a_{i j}{ }^{m} a_{k l m}
$$

for the hypersurface in Blaschke normal form (2.1). Under the coördinate freedom (2.2) this covariant transforms as a tensor under $\mathrm{GL}(3, \mathbb{C})$ and by scaling $S_{i j k l} \mapsto$ $r^{-1} S_{i j k l}$ under $w \mapsto r w$. The same is true of the quartic coefficients $b_{i j k l}$ of a Blaschke normal form. For the normal form A3 it is clear that $b_{i j k l}=9 \alpha S_{i j k l}$. Therefore, the same identity must hold in any Blaschke coördinates. In particular, this determines $\alpha$ in a manifestly affine invariant fashion.

From the point of view of normal forms, the classification in Bianchi class A is now complete. However, finding an explicit defining equation $w=F(x, y, z)$ for each hypersurface is a different matter. As explained in [1] for the case of surfaces in $\mathbb{C}^{3}$, the interpretation of the matrices $P, Q, R$ in Theorem 2 as generators of the symmetry algebra implies that the entire power series $F(x, y, z)$ may be defined implicitly by

$$
\left(\exp \left[\begin{array}{ccccc}
r P+s Q+t R & s \\
& t \\
& & & & 0 \\
0 & 0 & 0 & 0 & 0
\end{array}\right]\right)\left[\begin{array}{l}
0 \\
0 \\
0 \\
0 \\
1
\end{array}\right]=\left[\begin{array}{c}
x \\
y \\
z \\
F(x, y, z) \\
1
\end{array}\right] .
$$

Finding $F(x, y, z)$ by this means can be quite hard and involves a certain amount of trial and error. An example is presented in Appendix IV. Fortunately, once they are found there is no need to know where they came from: to confirm that they are correct is easily accomplished with computer algebra as follows.

- Apply Theorem 2 with $f(x, y, z)=\operatorname{Tr}^{N} F(x, y, z)$ for sufficiently large $N$ (say $N=5$ ), at the same time imposing (3.3), to find the generators $P, Q, R$ of the equiaffine symmetry algebra.

- Now check that (3.1) holds for $F(x, y, z)$ without truncation for these particular $P, Q, R$. This implies that the hypersurface defined by $F(x, y, z)$ is equiaffine homogeneous.

- Verify that the symmetry algebra is Bianchi class A, calculate the invariants $I, J, K, L$ and covariant $S_{i j k l}$, and refer to table (6.1) to locate the hypersurface in our classification.

Finally, we deal with the class B hypersurfaces of Theorem 5 . The values of the 
invariants of Appendix II on these hypersurfaces are as follows:-

\begin{tabular}{|l|l|l|}
\cline { 2 - 4 } \multicolumn{1}{c|}{} & Cubic Invariants & Remarks \\
\hline B1 & $I=J=K=L=0$ & Segre [111] \\
\hline B2 & $j=\frac{11}{25} \quad k=\frac{29}{125} \quad l=\frac{32}{3125}$ & Segre [111] \\
\hline B3 & $j=\frac{131}{289} \quad k=\frac{1073}{4913} \quad l=-\frac{864}{1419857}$ & Segre [111] \\
\hline B4 & $j=\frac{73}{200} \quad k=\frac{71}{500} \quad l=-\frac{27}{200000}$ & Segre [111] \\
\hline B5 & $j=\frac{11}{25} \quad k=\frac{29}{125} \quad l=\frac{32}{3125}$ & Segre [21] \\
\hline B6 & $j=\frac{11}{25} \quad k=\frac{29}{125} \quad l=\frac{32}{3125}$ & Segre [21] \\
\hline B7 & $I=J=K=L=0$ & Segre [21] \\
\hline B8 & $I=J=K=L=0$ & Segre [3] \\
\hline B9 & $I=J=K=L=0$ & Segre [3] \\
\hline B10 & $I=J=K=L=0$ & Segre [3] \\
\hline B11 & $I=J=K=L=0$ & Segre [3] \\
\hline
\end{tabular}

The remarks in this table are directed at the Segre characteristic of $N G$, computed in any Blaschke normal coördinates. Recall that this is affine invariant. In case [111], therefore, the cubic invariants are sufficient to identify the hypersurface. The first difficulty is to distinguish between B5 and B6. For this it is sufficient to consider the covariant

$$
T_{i}{ }^{j} \equiv 3 a_{i k l} a^{j k l}-4 b_{i}{ }^{j}{ }_{k}{ }^{k} .
$$

Under the coördinate freedom (2.2) it transforms as an endomorphism under $\mathrm{GL}(3, \mathbb{C})$ and by scaling $T_{i}{ }^{j} \mapsto r T_{i}{ }^{j}$ under $w \mapsto r w$. A computation yields

$$
T_{i}{ }^{j}=\frac{1}{2}\left[\begin{array}{ccc}
0 & -5 & 0 \\
0 & 0 & 0 \\
0 & 0 & 15
\end{array}\right] \text { and } \frac{1}{2}\left[\begin{array}{ccc}
0 & 0 & 0 \\
0 & 0 & 0 \\
0 & 0 & 15
\end{array}\right]
$$

for the normal forms of $\mathbf{B 5}$ and $\mathbf{B 6}$, respectively. Notice that the rank of $T_{i}{ }^{j}$ is 2 for B5 but 1 for B6. This holds in any Blaschke coördinates and provides an easily computable affine invariant distinction.

Now we must deal with $\mathbf{B} 8-\mathbf{B 1 1}$. The pair $\mathbf{B 8}$ and $\mathbf{B 9}$ are already affinely distinct from $\mathbf{B 1 0}$ and $\mathbf{B 1 1}$ at the cubic level:-

$$
2 x y+z^{2}+x^{3}+x^{2} y-2 x z^{2} \text { versus } 2 x y+z^{2}+x^{2} y-2 x z^{2} .
$$

An easily implemented distinction is that the second of these admits an affine symmetry (5.2) fixing the origin. Infinitesimally, this corresponds to being an eigenfunction for a vector field of the form $X^{i}{ }_{j} u^{j} \partial / \partial u^{i}$. The pair $\mathbf{B} 8$ and $\mathbf{B 1 0}$ are distinguished from $\mathbf{B 9}$ and $\mathbf{B 1 1}$ by the vanishing of the covariant

$$
U_{i}{ }^{j} \equiv a_{i k l} a^{j k l}-8 b_{i}{ }^{j}{ }^{k} .
$$

The upshot of this discussion is that the proof of Theorem 1 is reduced to computation. Indeed, the identification of any equiaffine homogeneous hypersurface in the classification lists of Theorems 4 and 5 , is a matter of computation and can be completely automated. Such an automation is described in Appendix III. This proves Theorem 1. 
Appendix I. Trace-free Cubics. It is convenient to use the 2-1 homomorphism of Lie groups

$$
\mathrm{SL}(2, \mathbb{C}) \longrightarrow \mathrm{SO}(3, \mathbb{C})
$$

as detailed in [7]. Specifically, if $\operatorname{SL}(2, \mathbb{C})$ is acting on $\mathbb{C}^{2}$ by matrix multiplication

$$
\left[\begin{array}{l}
s \\
t
\end{array}\right] \mapsto\left[\begin{array}{ll}
a & b \\
c & d
\end{array}\right]\left[\begin{array}{l}
s \\
t
\end{array}\right]
$$

then the induced action on $\mathbb{C}^{3}$

$$
\left[\begin{array}{l}
x \\
y \\
z
\end{array}\right] \mapsto\left[\begin{array}{ccc}
a^{2} & -b^{2} / 2 & -a b \\
-2 c^{2} & d^{2} & 2 c d \\
-2 a c & b d & a d+b c
\end{array}\right]\left[\begin{array}{l}
x \\
y \\
z
\end{array}\right]
$$

preserves our quadratic form (2.3). The direct link is to associate

$$
s^{2} \leftrightarrow-2 x \quad \text { st } \leftrightarrow z \quad t^{2} \leftrightarrow y,
$$

identifying the quadratics in $s, t$ with $\mathbb{C}^{3}$. In a similar vein, the action of $\mathrm{SL}(2, \mathbb{C})$ on binary sextics induces the action of $\mathrm{SO}(3, \mathbb{C})$ on trace-free cubic polynomials in $x, y, z$ under the association

$$
\begin{array}{ccc}
s^{6} & \leftrightarrow & -8 x^{3} \\
s^{5} t & \leftrightarrow & 4 x^{2} z \\
s^{4} t^{2} & \leftrightarrow & 4\left(x^{2} y-2 x z^{2}\right) / 5 \\
s^{3} t^{3} & \leftrightarrow & -2\left(3 x y z-z^{3}\right) / 5 \\
s^{2} t^{4} & \leftrightarrow & -\left(2 x y^{2}-2 y z^{2}\right) / 5 \\
s t^{5} & \leftrightarrow & y^{2} z \\
t^{6} & \leftrightarrow & y^{3} .
\end{array}
$$

Normalizing a sextic also normalizes the corresponding trace-free cubic. The table of normal forms (2.5) is now straightforward. In the generic case, a sextic polynomial has two isolated zeroes which may be placed at 0 and $\infty$ by a suitable Möbius transformation (Appendix I.1). The necessarily non-zero coefficients of $s^{5} t$ and $s t^{5}$ may be set equal by

$$
s \mapsto \lambda s \quad t \mapsto \lambda^{-1} t
$$

for suitable $\lambda$ and then rescaled to unity with (2.2). This gives normal form \#1 in (2.5). At the other extreme, normal form \#10 arises when the sextic has just one 6 -fold zero.

Appendix II. Classical Invariants. A further consequence of identifying trace-free cubics in 3 dimensions with binary sextics is that the classical invariant theory of the sextic may be carried over (compare [8, Chapter 8]). In [4], generators of the ring of invariants are determined. They are of degree 2, 4, 6, 10, and 15. However, it is only the invariants of even degree that extend from $\mathrm{SO}(3, \mathbb{C})$ to $\mathrm{O}(3, \mathbb{C})$ so, for our purposes, the invariant of degree 15 may be discarded. Here are the invariants of degree 2 and $4:-$

$$
I=a_{i j k} a^{i j k} \quad J=a_{i j k} a^{i j l} a_{l m n} a^{m n i}
$$


A more convenient notation is to draw a circle for each copy of $a_{i j k}$ and to wire them up as in accordance with how they are to be contracted (see [7]). Here are the four generators using this notation:-
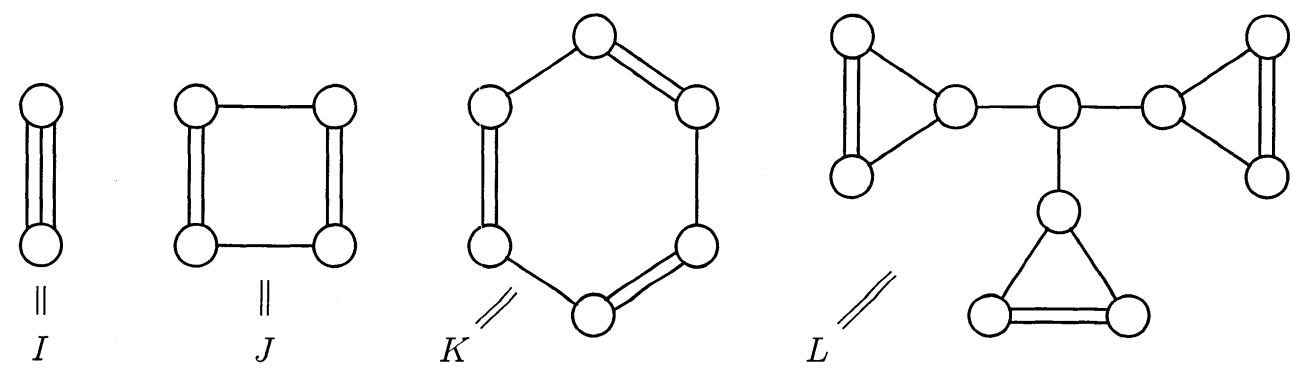

They freely generate the ring of all invariants. Local affine invariants are induced as follows. Writing a hypersurface in Blaschke normal form (2.1), we may compute $I, J, K, L$ from its cubic terms $a_{i j k}$ using the quadratic form $g_{i j}$ to raise indices. Under the remaining coördinate freedom (2.2), these quantities simply scale:

$$
I \mapsto r I \quad J \mapsto r^{2} \quad K \mapsto r^{3} K \quad L \mapsto r^{5} L .
$$

Thus, when $I \neq 0$, the quotients

$$
j \equiv J / I^{2} \quad k \equiv K / I^{3} \quad l \equiv L / I^{5}
$$

are absolute affine invariants.

Appendix III. Computer Programs. The following programs are written for the computer algebra system MAPLE.

$\begin{array}{llll}\text { defns } & \text { classAcase1 } & \text { classB } & \text { list } \\ \text { makeeqns } & \text { classAcase2 } & \text { classBsegre111 } & \text { invariants } \\ \text { classA } & \text { classAcase3 } & \text { classBsegre21type1 } & \text { locate } \\ \text { changecoords } & \text { classAcase4 } & \text { classBsegre21type2 } & \\ \text { classAcontd } & \text { classAcase5 } & \text { classBsegre3 } & \end{array}$

They are available electronically. The following two locations

- ftp://ftp.maths.adelaide.edu.au/pure/meastwood/maple/ea

- ftp://ftp.maths.adelaide.edu.au/pure/meastwood/maple6/ea

contain these programs for MAPLE V and MAPLE 6, respectively. They perform the following tasks.

defns This program sets the scene by introducing the $4 \times 4$ matrices $P, Q, R$, making useful changes of variables, and specifying $f(x, y, z)$, a cubic polynomial without constant or linear terms. The possibilities used in the article are already included in this file. The results are saved in a file PQRf.m (in MAPLE internal format).

makeeqns This program makes the equations that must be solved, as discussed in Section 3. The program 'defns' must be run first in order to specify $f(x, y, z)$, a polynomial of degree 3 without constant or linear terms. The linear equations (3.1) and (3.3) are formed and solved. Then the equations (3.4) in the variables (3.9) are formed and stored in a file called eqns.m (in MAPLE internal format).

classA This program solves the equations (3.4) arising from the ten normalizations of Appendix I, assuming that the symmetry algebra is Bianchi class A. See Section 4 for discussion.

changecoords This program checks that the coördinate changes in Section 4 do as is claimed. 
classAcontd This program takes one of the five final normalizations derived in Section 4 and again solves the resulting equations (3.4).

classAcase 1-5 In each case, the solutions from 'classAcontd' are used to specify the generators $P, Q, R$ of the corresponding symmetry algebra and, by using Theorem 2 with $N=4$, the quartic terms in the defining equation are found.

classB This program solves the system of equations arising from Blaschke normal coördinates together with the normalizations in Bianchi class B as expounded in Section 5 .

classBsegre111-3 These programs complete the analysis of Bianchi class B. In each case, generators of the symmetry are displayed and higher order terms in the defining equation are computed.

list This file contains the final list of normal forms, as in Theorems 4 and 5 . The program itself finds the equiaffine symmetries of any given hypersurface and determines whether the symmetry algebra closes.

invariants This program computes the classical cubic invariants of a nondegenerate hypersurface, assumed to be in Blaschke normal form. The invariants are defined in Appendix II and used in Section 6. Also in Section 6 are covariants and other devices used in distinguishing between various hypersurfaces. These are all computed by this program.

locate This program finds the symmetries of a hypersurface given in closed form and determines whether it is equiaffine homogeneous. It says if there is equiaffine isotropy. For equiaffine homogeneous hypersurfaces without isotropy, it expands the defining function to order 4 and changes to Blaschke normal form. Then it uses Section 6 to locate the hypersurface in the list A1-B11. The hypersurfaces of Theorem 1 are included in this file. In particular, bearing in mind the discussion of Section 6, the successful running of this program in these cases, proves Theorem 1.

Appendix IV. Exponentation of a Symmetry Algebra. Consider the hypersurface A3 from Theorem 4. It is convenient to rescale: $w \mapsto(8 / 27) w, x \mapsto(4 / 3) x$, $y \mapsto(4 / 3) y, z \mapsto \sqrt{32 / 27} z$. Then $\mathbf{A 3}$ is defined by the power series

$$
w=12 x y+4 z^{2}+8 x^{3}+8 y^{3}+15 x^{2} y^{2}+8 x^{3} z-8 y^{3} z-6 x y z^{2}-z^{4}+\mathrm{O}(5) .
$$

Theorem 2 yields $P, Q, R$ as follows:-

$$
\left[\begin{array}{cccc}
0 & 0 & -1 & 1 / 8 \\
-2 & 0 & 0 & 0 \\
0 & 3 / 2 & 0 & 0 \\
0 & 12 & 0 & 0
\end{array}\right],\left[\begin{array}{cccc}
0 & -2 & 0 & 0 \\
0 & 0 & 1 & 1 / 8 \\
-3 / 2 & 0 & 0 & 0 \\
12 & 0 & 0 & 0
\end{array}\right],\left[\begin{array}{cccc}
-1 / 3 & 0 & 0 & 0 \\
0 & 1 / 3 & 0 & 0 \\
0 & 0 & 0 & 1 / 8 \\
0 & 0 & 8 & 0
\end{array}\right]
$$

Before substituting into (6.2), it is convenient to conjugate by

$$
\left[\begin{array}{cccc}
1 & 0 & 0 & 0 \\
0 & 1 & 0 & 0 \\
0 & 0 & 8 & 1 \\
0 & 0 & 8 & -1
\end{array}\right]
$$


Then (6.2) asks us to calculate $\exp (r \hat{P}+s \hat{Q}+t \hat{R})$ where

$$
\begin{gathered}
\hat{P}=\left[\begin{array}{ccccc}
0 & 0 & 0 & -1 / 8 & 1 \\
-2 & 0 & 0 & 0 & 0 \\
0 & 24 & 0 & 0 & 0 \\
0 & 0 & 0 & 0 & 0 \\
0 & 0 & 0 & 0 & 0
\end{array}\right] \quad \hat{Q}=\left[\begin{array}{ccccc}
0 & -2 & 0 & 0 & 0 \\
0 & 0 & 1 / 8 & 0 & 1 \\
0 & 0 & 0 & 0 & 0 \\
-24 & 0 & 0 & 0 & 0 \\
0 & 0 & 0 & 0 & 0
\end{array}\right] \\
\hat{R}=\left[\begin{array}{ccccc}
-1 / 3 & 0 & 0 & 0 & 0 \\
0 & 1 / 3 & 0 & 0 & 0 \\
0 & 0 & 1 & 0 & 8 \\
0 & 0 & 0 & -1 & 8 \\
0 & 0 & 0 & 0 & 0
\end{array}\right]
\end{gathered}
$$

This is possible but much easier is to calculate $\exp (r \hat{P}) \exp (s \hat{Q}) \exp (t \hat{R})$. Since the symmetry algebra is closed:

$$
[\hat{Q}, \hat{R}]=\frac{2}{3} \hat{Q} \quad[\hat{R}, \hat{P}]=\frac{2}{3} \hat{P} \quad[\hat{P}, \hat{Q}]=3 \hat{R}
$$

this gives a different parametrization of the same hypersurface. We find

$$
\exp (r \hat{P}) \exp (s \hat{Q}) \exp (t \hat{R})\left[\begin{array}{l}
0 \\
0 \\
0 \\
0 \\
1
\end{array}\right]=\left[\begin{array}{c}
x \\
y \\
z \\
w \\
1
\end{array}\right]
$$

where

$$
\begin{aligned}
w & =8 s^{3} e^{t}-8 e^{-t}+8 \\
x & =-s^{2} e^{t}-r s^{3} e^{t}+r e^{-t} \\
y & =s e^{t}+2 r s^{2} e^{t}+r^{2} s^{3} e^{t}-r^{2} e^{-t} \\
z & =8 e^{t}+24 r s e^{t}+24 r^{2} s^{2} e^{t}+8 r^{3} s^{3} e^{t}-8 r^{3} e^{-t}-8 .
\end{aligned}
$$

If we make the affine change of variables

$$
W=(w-8) / 8 \quad X=x \quad Y=y \quad Z=(z+8) / 8,
$$

and notice that $s=X^{2}-W Y$, then

$$
\begin{aligned}
X & =-s^{2} \lambda-r W \\
Y & =(1+2 r s) s \lambda+r^{2} W \\
Z & =\left(1+3 r s+3 r^{2} s^{2}\right) \lambda+r^{3} W
\end{aligned}
$$

where $\lambda=e^{t}$ and $s=X^{2}-W Y$. If we use the first equation to eliminate $\lambda$ from $s Y$ and $s^{2} Z$, we obtain a quadratic equation and a cubic equation in $r$ with coefficients that are polynomials in $W, X, Y, Z$. The resultant of these equations is, therefore, a polynomial identity in $W, X, Y, Z$. A computation gives

$$
W\left(W Y-X^{2}\right)^{7}\left(6 W X Y Z-4 X^{3} Z-3 X^{2} Y^{2}+W^{2} Z^{2}+4 W Y^{3}-1\right)
$$

as the irreducible decomposition of this resultant. The basepoint of this hypersurface is the origin in $(w, x, y, z)$ coördinates. This corresponds to $(W, X, Y, Z)=(-1,0,0,1)$. Therefore,

$$
6 W X Y Z-4 X^{3} Z-3 X^{2} Y^{2}+W^{2} Z^{2}+4 W Y^{3}=1
$$

provides an explicit defining function near $(-1,0,0,1)$. If preferred, we can replace $W \mapsto-W, Y \mapsto-Y$, to obtain $(1,0,0,1)$ as basepoint. 


\section{REFERENCES}

[1] M.G. EASTwOOD AND V.V. EzHov, On affine normal forms and a classification of homogeneous surfaces in affine three-space, Geom. Dedicata, 77 (1999), pp. 11-69.

[2] M.G. EAstwood AND V.V. Ezhov, Classifying the homogeneous hypersurfaces in a homogeneous space, in Colloque de Géométrie Complexe (eds. F. Norguet and S. Ofman), International Press, to appear.

[3] M.G. EAstwood AND V.V. EzHov, Homogeneous hypersurfaces with isotropy in affine fourspace, Tr. Mat. Inst. Steklova, to appear.

[4] E.B. Elliott, Algebra of Quantics, Clarendon Press 1895.

[5] H. Guggenheimer, Differential Geometry, McGraw-Hill 1963.

[6] K. LEICHTWEISS, Über eine geometrische Deutung des Affinnormalenvektors einseitig gekrümmter Hyperflächen, Arch. Math., 53 (1989), pp. 613-621.

[7] R. Penrose and W. Rindler, Spinors and Space-time, vol. 1, Cambridge University Press, 1984.

[8] R. Penrose And W. Rindler, Spinors and Space-time, vol. 2, Cambridge University Press, 1986.

[9] K. NOMIZU AND T. SASAKI, A new model of unimodular-affinely homogeneous surfaces, Manuscripta Math., 73 (1991), pp. 39-44.

[10] K. Nomizu and T. SASAKI, Affine Differential Geometry, Cambridge University Press, 1994.

[11] H.W. Turnbull And A.C. Artken, An Introduction to the Theory of Canonical Matrices, Blackie and Son, 1932. 
